\title{
Association of a hi-tech with a bio-tech technique in the treatment of early osteoarthritis of the knee: a case report
}

\author{
FRANCESCO MATTIA UBOLDI ${ }^{1}$, PAOLO FERRUA ${ }^{1}$, ANDREA PARENTE ${ }^{2}$, \\ STEFANO PASQUALOTTO ${ }^{2}$, MASSIMO BERRUTO ${ }^{1}$
}

${ }^{1}$ Knee Surgery Department, Gaetano Pini Hospital, Milan, Italy
${ }^{2}$ Department of Orthopedics and Traumatology, University of Milan, Italy

\begin{abstract}
Meniscal replacement to treat early osteoarthritis of the knee after meniscectomy may be accompanied by other surgical procedures to treat factors predisposing to a negative intervention outcome. Overload of the medial compartment in slight varus can be reduced by applying the new KineSpring system, which can promote the best possible outcome of a biodegradable meniscal scaffold implantation, without producing biomechanical and anatomical alterations of the joint. This is the first case report on the combination of these hi-tech and bio-tech techniques.
\end{abstract}

Keywords: KineSpring, knee, meniscus, osteoarthritis, scaffold.

\section{Case presentation}

In May 2013 a 43-year-old man was admitted with persistent medial right knee pain after a double partial meniscectomy (procedures performed in 2011 and 2012). A conservative treatment had been performed without success. On clinical evaluation the knee was not swollen and the range of motion (ROM) was complete. Pain was scored as $7 / 10$ on a visual analog scale (VAS), and was worsened by acupressure on the middle and posterior third of the medial joint line. No

\section{Corresponding Author:}

Francesco Mattia Uboldi, MD

Knee Surgery Department, Gaetano Pini Hospital

P.zza Cardinal Ferrari 1, 20122 Milan, Italy

E-mail: fraubo@gmail.com pathological ligament laxity was observed. The patient also reported experiencing pain in daily activities. Imaging demonstrated minimal signal alteration of the medial femoral condyle rim, with minimal osteophytosis on the medial tibial plateau and narrowing of the medial articular joint line, consistent with grade 2 osteoarthritis (OA) according to the Kellegren-Lawrence classification (1). Long standing X-rays showed a varus alignment of three degrees that did not change in single stance (Fig. 1).

Treatment was performed using an actifit meniscal scaffold (Orteq, London, UK); in addition, given that the minimal varus alignment constituted a contraindication to high tibial osteotomy, a KineSpring System (Moximed, Hayward, CA, USA) was implanted in order to unload the medial compartment and reduce the overload on the meniscal scaffold.

Once arthroscopic evaluation had confirmed the good quality and stability of the remaining meniscus, the scaffold implant site in the residual meniscus was prepared. The Actifit biodegradable polyurethane scaffold was implanted in the residual meniscus and fixed with four all-inside sutures (FasT-Fix, Smith \& Nephew, Andover, MA, USA).

The arthroscopic procedure was complete after evaluation of the implant stability with 20 full passive ROM cycles (Fig. 2).

The KineSpring implantation was then performed by first making two $5 \mathrm{~cm}$ oblique incisions on the medial side of the femoral epicondyle and proximal medial tibial metaphysis.

The KineSpring device comprises a subcutaneous shock absorber supported by two plates fixed medially on the femoral epicondyle and on the medial tibial metaphysis. As first step, mandatory for the correct 


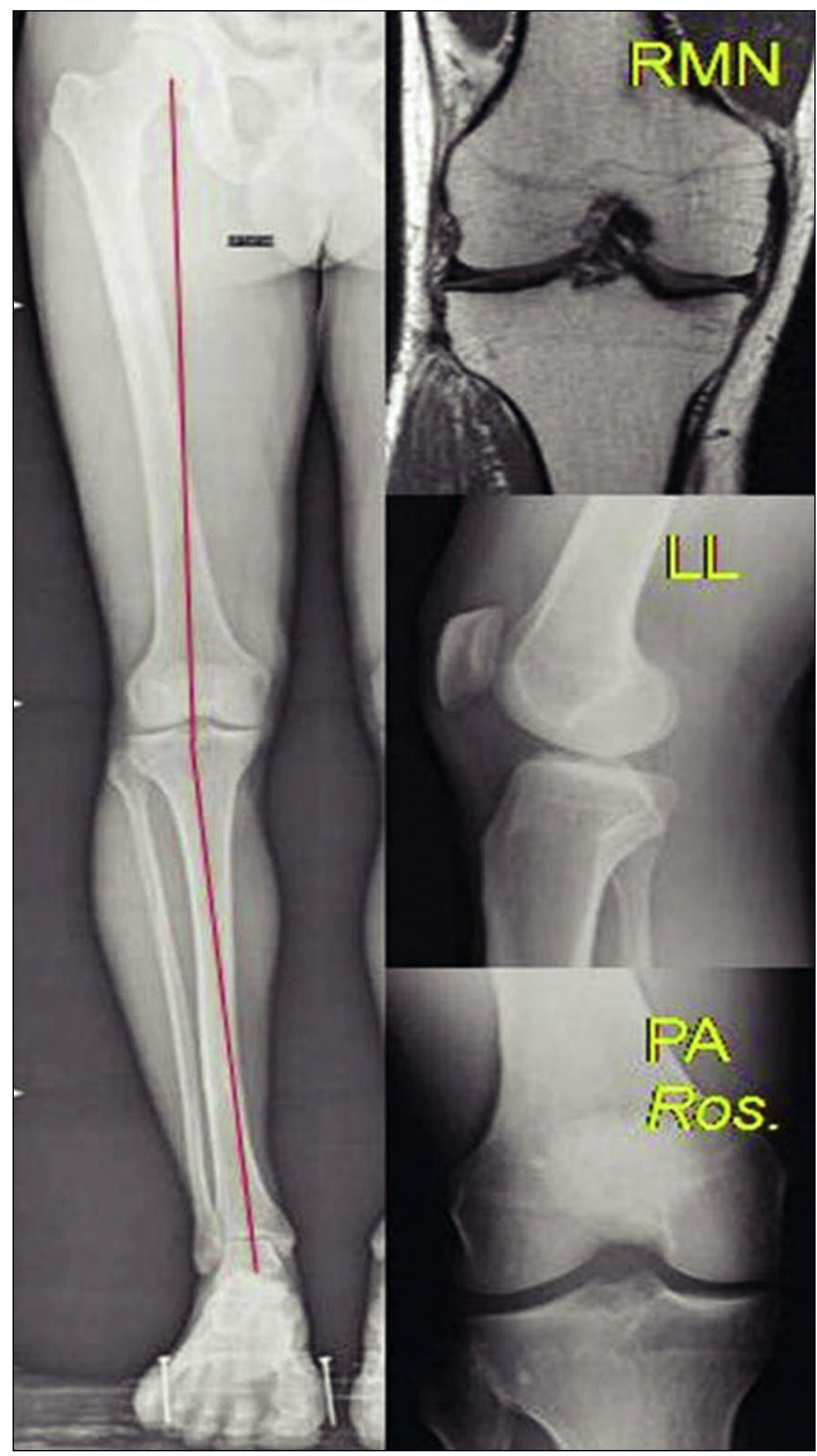

Fig. 1. X-ray and MRI images: knee early osteoarthritis as an outcome of partial media meniscectomy. positioning of the implant, the femoral starting point was identified using fluoroscopy (Fig. 3). Using this point of reference the femoral plate was fixed with one lag screw and three angular stability screws. Then, the tibial plate was inserted behind the pes anserinus. A blunt dissection was performed to create a subcutaneous path between the two incisions, in which the shock absorber was implanted.

The system is stabilized in varus stress position at $10^{\circ}$ of flexion and the shock absorber was activated bringing the knee back to the relaxed position.

The system can bear a $13 \mathrm{~kg}$ load during walking, opening the medial articular space by $3 \mathrm{~mm}$ in the unloading position (Fig. 4) (2). Particular care should be taken during skin closure to avoid wound dehiscence.

Postoperatively, partial weight-bearing with crutches was allowed for six weeks. Passive ROM with continuous passive motion was started three days after surgery, with the aim of reaching full ROM in 6-8 weeks avoiding wound traction. Squatting and pivoting were forbidden for three months to protect the meniscal scaffold healing process.

At the eight-month follow-up a significant improvement was noted in all evaluations: pain had decreased from 6 to 1 on the VAS scale, the Lysholm score had improved from 60 to 92 points and the subjective IKDC score from 57 to 82 points. At the two-year follow-up, pain was rated as absent (VAS $=0$ ), the Lysholm score was 99 points and the IKDC score was 92 points. The patient was completely satisfied with the results. 


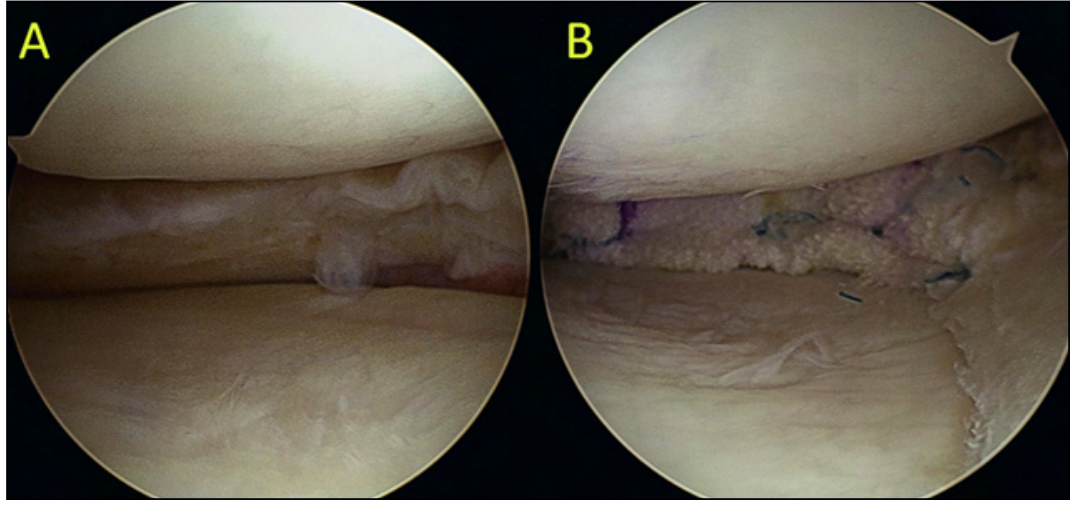

Fig. 2. Arthroscopic images. A. Good medial meniscus wall availability. B. Actifit ${ }^{\circledR}$ meniscal scaffold implants with all-inside sutures.

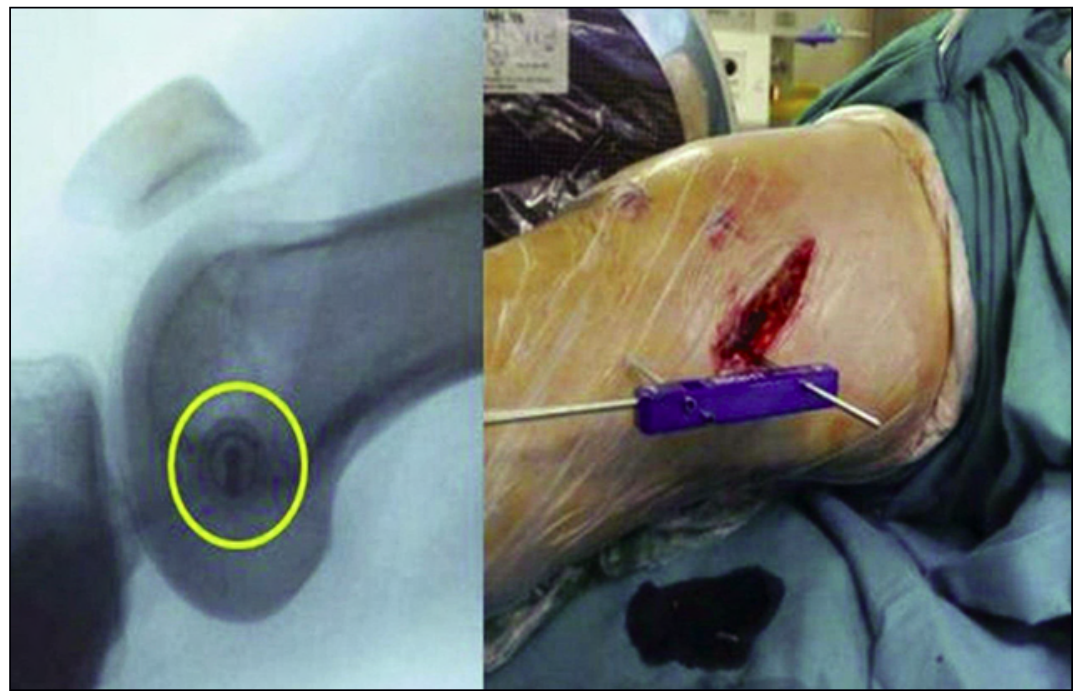

Fig. 3. Fluoroscopic identification of the joint rotation center and positioning of the KineSpring femoral guide.

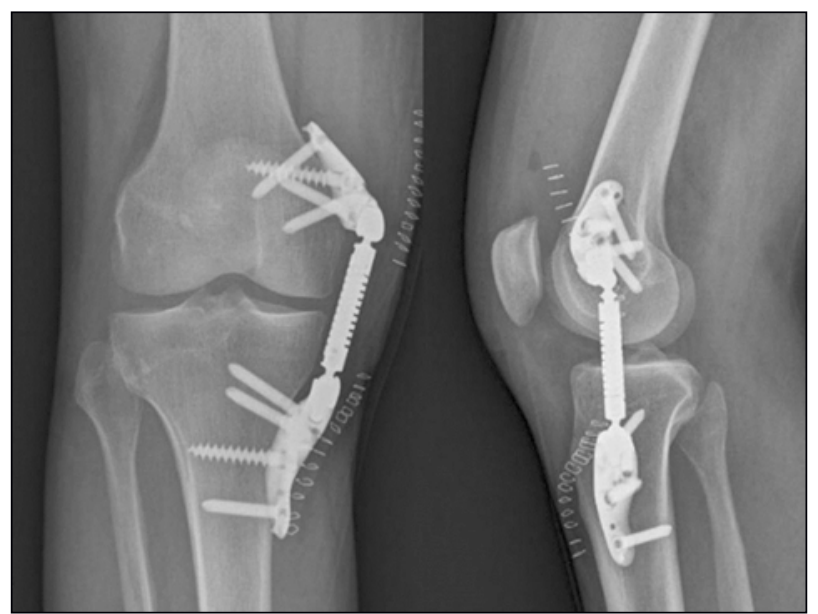

Fig. 4. Post-operative X-ray images: the KineSpring system in place.

\section{Discussion}

This clinical case highlights the possibility of combining the use of a meniscal prosthesis with the implantation of a KineSpring system in the treatment of residual medial pain after a medial meniscectomy.

A patient submitted to partial meniscectomy, even if there was no varus or valgus deformity at the time of surgery, has a probability of between 46 and $89 \%$ of developing early OA in the 10 years after surgery, and a varus deformity develops in $60 \%$ of patients after a medial meniscectomy (3). Meniscal scaffolds are considered a valid option to restore meniscal function and prevent osteoarthritic evolution, with successful results reported at short- and medium-term follow-up (4). In an experimental study, polyurethane meniscal scaffolds were used to enhance cellular migration from the meniscal wall and to allow regeneration of tissue biomechanically similar to native meniscus (5); at five years after implantation the porous structure of such scaffolds has been reported to be well filled out with an amorphous and heterogeneous matrix surrounding fibrochondrocytes $(6,7)$. In the reported case, the presence of a minimal varus $\left(3^{\circ}\right)$ was not considered a contraindication for this technique although it can be a negative prognostic factor for the final result. To obtain partial unloading of the medial compartment the main alternative treatment in adult patients is high tibial osteotomy $(8,9)$. In this case, since the varus was minimal, the KineSpring system was considered the optimal solution, as it unloads medial compartment without definitively altering the knee anatomy, given that the implant is totally reversible. The short- 


\section{Joints J}

term result in our patient seems to confirm this surgical choice, but further follow-up and further studies are needed in order to validate this association as a valid option for preventing post-meniscectomy OA.

\section{References}

1. Kellgren JH, Jeffrey M, Ball J. Atlas of Standard Radiographs. Blackwell Scientific, Oxford. 1963; Vol 2.

2. Waller C, Hayes D, Block JE, London NJ. Unload it: the key to the treatment of knee osteoarthritis. Knee Surg Sports Traumatol Arthrosc. 2011;19:1823-1829.

3. Allen PR, Denham RA, Swan AV. Late degenerative changes after meniscectomy. Factors affecting the knee after operation. J Bone J Surg Br. 1984;66:666-671.

4. Bouyarmane H, Beaufils P, Bellemans J, et al. Clinical results of polyurethane scaffold in lateral meniscus segmental defects. Arthroscopy. 2013;29:e51-e52.
5. Maher SA, Rodeo SA, Doty SB, et al. Evaluation of a porous polyurethane scaffold in a partial meniscal defect ovine model. Arthroscopy. 2010;26:1510-1519.

6. Bulgheroni P, Bulgheroni E, Regazzola G, et al. Polyurethane scaffold for the treatment of partial meniscal tears. Clinical results with a minimum two-year follow-up. Joints. 2013; 1:161-166.

7. Kon E, Filardo G, Zaffagnini S, et al. Biodegradable polyurethane meniscal scaffold for isolated partial lesions or as combined procedure for knees with multiple comorbidities: clinical results at 2 years. Knee Surg Sports Traumatol Arthrosc. 2014;22:128-134.

8. Robinson PM, Papanna MC, Somanchi BV, et al. High tibial osteotomy in medial compartment osteoarthritis and varus deformity using the Taylor spatial frame: early results. Strategies Trauma Limb Reconstr. 2011;6:137-145.

9. Harris JD, McNeilan R, Siston RA, et al. Survival and clinical outcome of isolated high tibial osteotomy and combined biological knee reconstruction. The Knee. 2013;20:154-161. 